\title{
Exploration au champ et interprétation de la variation horizontale et verticale de la résistance stomatique : cas d'une culture de luzerne (Medicago sativa L.)
}

\author{
Nader KATERJI, Alain PERRIER $\left({ }^{*}\right)$ \& Abdel Krim OULID-AÏSSA $\left({ }^{*}\right)$ \\ I.N.R.A., Station de Bioclimatologie, route de Saint-Cyr, F 78000 Versailles \\ (*) Laboratoire de recherche de la Chaire de Bioclimatologie de l'l.N.A.-P.G., 16, rue Claude-Bernard, \\ F 75231 Paris Cedex 05
}

RÉSUMÉ

\begin{abstract}
Une étude réalisée sur une culture de luzerne au champ a permis d'analyser les problèmes de la répartition et de la représentativité des mesures de résistance stomatique $\left(r_{s}\right)$ à différentes échelles d'espace (feuille, strate, plante entière). Les résultats obtenus au cours de cette étude ont permis de dégager quelques faits importants :

- La variation spatiale des mesures de résistance stomatique obtenues sur des feuilles physiologiquement stables (même stade de développement ou situées dans la même strate) dépend des conditions climatiques auxquelles sont soumises les feuilles testées; plus les conditions sont stables, plus cette variabilité est faible. - A l'échelle de la plante entière, il apparaît que les valeurs élevées et très dispersées de la résistance stomatique $r_{\mathrm{s}}$ observées au fur et à mesure que l'on s'éloigne du sommet de la végétation sont la conséquence d'une diminution progressive et hétérogène de l'éclairement.

Pour interpréter les résultats obtenus, les auteurs ont examiné plusieurs hypothèses dont certaines ont reçu des confirmations expérimentales.
\end{abstract}

Mot clé additionnel : Hétérogénéité spatiale.

Assessment and interpretation of horizontal and vertical variation in stomatal diffusion resistance in lucerne in the field A study carried out on a lucerne crop under field conditions made in possible to analyse the distribution and
representativity of stomatal diffusion resistance $\left(\mathrm{r}_{\mathrm{s}}\right)$ measures at various spatial levels (leaf, layer, whole
plant). The results obtained during this study led to several important conclusions : 1) The spatial variation in
stomatal diffusion resistance measures obtained from physiologically stable leaves (same stage of develop-
ment, or located in the same layer) depended upon the climatic conditions to which the tested leaves were
exposed ; the more stable the conditions, the lesser the degree of variation. 2) On a whole plant scale, it
appeared that the high and very dispersed values of stomatal diffusion resistance $\mathrm{r}_{\mathrm{s}}$ observed the further one
moves away from the plant tops were the consequence of a progressive and heterogeneous decrease in the
amount of light.
In order to interpret the results obtained, the authors examine different hypotheses, several of which have
received experimental verification.

Additional key word : Spatial heterogeneity.

\section{INTRODUCTION}

En dépit des multiples études dont il a fait et fait actuellement l'objet, le mécanisme de régulation stomatique n'est que très partiellement cerné en milieu naturel. En effet, les résultats présentés dans la littérature (BERGER, 1971 ; DJAVANCHIR, 1971 ; CATSKY et al., 1973) concernent toujours des mesures ponctuelles particulières obtenues avec certaines précautions (on choisit par exemple unique- ment les feuilles du sommet; on suit l'évolution des stomates d'un même emplacement, etc...). En fait, la distribution réelle de ces quelques situations particulières où des mesures directes ont été faites ainsi que l'importance de ces cas par rapport à l'ensemble des situations au sein d'un couvert n'ont jamais été réellement abordées. Il est frappant de constater que, dans les nombreux ouvrages qui traitent du problème de l'eau et dans lesquels les chapitres consacrés à la résistance stomatique occupent une place 
importante (HEATH, 1959 ; STOCKER, 1961 ; DE PARCEVAUX, 1963 ; SLATYER, 1967 ; MEIDNER \& MANSFIELD, 1968 ; KozlowsKI, 1968, 1972, 1976 ; Cowan, 1977), on ne trouve pas de référence sur l'analyse de ces répartitions et sur les problèmes d'échantillonnage que pose cette analyse.

L'objet de cet article est d'arriver à une meilleure connaissance de la répartition des valeurs de résistance stomatique $r_{s}$ au sein d'un couvert et des interprétations qu'il est possible d'en donner. D'une façon plus précise, les questions auxquelles notre étude doit pouvoir répondre sont les suivantes:

- Quelle est la représentativité d'une mesure ponctuelle de résistance stomatique en fonction des conditions climatiques précises au point de mesure?

- Dans quelles conditions y a-t-il une tendance à l'homogénéité dans l'ouverture des stomates et dans quelles conditions a-t-on, au contraire, une distribution dispersée ? Comment peut-on expliquer ces tendances?

- Quelle est la distribution des valeurs de résistance stomatique à l'intérieur d'un couvert végétal et comment peut-on interpréter cette distribution?

Pour répondre à ces questions, nous commencerons d'abord par analyser à l'échelle de la feuille l'hétérogénéité entre les différentes mesures de $r_{s}$ sur divers points de celleci, puis nous étudierons la variation entre les différentes mesures de $r_{s}$ sur des feuilles physiologiquement stables et soumises à des conditions climatiques relativement constantes. Enfin nous analyserons l'hétérogénéité observée dans les mesures de $r_{s}$ réalisées au sein d'une même tranche de végétation sur des feuilles soumises à des conditions climatiques et physiologiques très variables et comparerons les différentes tranches entre elles.

\section{DISPOSITIF EXPÉRIMENTAL}

Les expériences ont été réalisées au cours des années 1978-79 et 80 au domaine expérimental de La Minière (Yvelines) sur un couvert de luzerne (variété « Du Puits») qui se trouve dans des conditions hydriques non limitantes (KATERJI, 1982). Le choix de la culture a été dicté par la croissance rapide de la luzerne qui permet d'étudier au cours de l'année plusieurs cycles de végétation dans des conditions climatiques différentes. Pour rester dans des conditions phénologiques voisines, la fauche de la culture a été effectuée systématiquement à l'apparition des premiers boutons floraux; ainsi l'étude entreprise concerne seulement le stade correspondant à la croissance végétative (DEMARLY, 1957). Les mesures de résistance stomatique ont été obtenues par le poromètre à diffusion de vapeur d'eau (Automatic porometer MK II) dont le principe et la fiabilité au champ ont été décrits dans un autre article (KATERJI \& OULID-AïsSA, 1983). Notons seulement que toutes les mesures de résistance stomatique ont été réalisées à l'aide d'un poromètre protégé qui permet de neutraliser au mieux les perturbations induites par les facteurs du milieu (vent, écart de température entre la feuille et la chambre de transpiration du poromètre).

\section{RÉSULTATS EXPÉRIMENTAUX}

\section{A. Résistance stomatique à l'échelle de la feuille : varia- tion et variabilité}

La figure 1 donne, à titre d'exemple, les différentes valeurs de $r_{s}$ obtenues pendant $15 \mathrm{mn}$ au cours d'une journée de béau temps sur la face supérieure de 5 feuilles de luzerne, composées chacune de 3 folioles. Ces feuilles ont été choisies au hasard sur 5 plantes; néanmoins toutes les feuilles avaient le même âge, la même inclinaison (parfaitement horizontales) et la même position sur la plante ( $1^{\text {re }}$ feuille en partant du haut). Ainsi les échantillons se trouvent dans des conditions d'ensoleillement identiques. On constate que les valeurs de $r_{s}$ mesurées sur les différentes folioles sont assez proches. Cependant l'hétérogénéité (c'est-à-dire la différence entre la plus petite et la plus grande mesure de $r_{s}$ sur la même feuille) est très variable d'une feuille à l'autre ; presque nulle dans certains cas, elle peut être parfois de l'ordre de 5 à 40 p. 100 . Les conditions climatiques étant homogènes sur les feuilles, cette hétérogénéité ne peut que refléter l'hétérogénéité biologique dans la réaction de la feuille ou l'erreur expérimentale de la mesure. En ce qui concerne l'hétérogénéité biologique, plusieurs raisons peuvent être invoquées; d'une part, la densité stomatique locale peut être différente ( $\mathrm{SCHOCH}$, 1972), d'autre part, il existe des variations cycliques dans les mouvements stomatiques pouvant entraîner des différences entre folioles. Rappelons en effet que, depuis plusieurs années, on a mis en évidence des mouvements stomatiques de type cyclique (oscillation) pour des conditions d'environnement constantes (BARRS, 1971 ; COWAN, 1972 ; JOHNSON, 1973 ; FARQUHAR \& COWAN, 1974 ; AHO, 1981) ; il semble toutefois que le comportement oscillatoire est aléatoire et que des plantes de même âge, ayant la même histoire et soumises aux mêmes environnements, ont montré des comportements oscillatoires très différents (CowAN, 1972). L'interprétation de ces oscillations a fait l'objet de nombreuses études récentes. En fait, il est impossible de se prononcer sur l'origine exacte de l'hétérogénéité obtenue dans les mesures de $r_{s}$ car elle paraît faible pour l'ensemble des feuilles mesurées et reste dans l'ordre de grandeur de l'erreur expérimentale (KATERII \& OULID-AISSA, 1983).
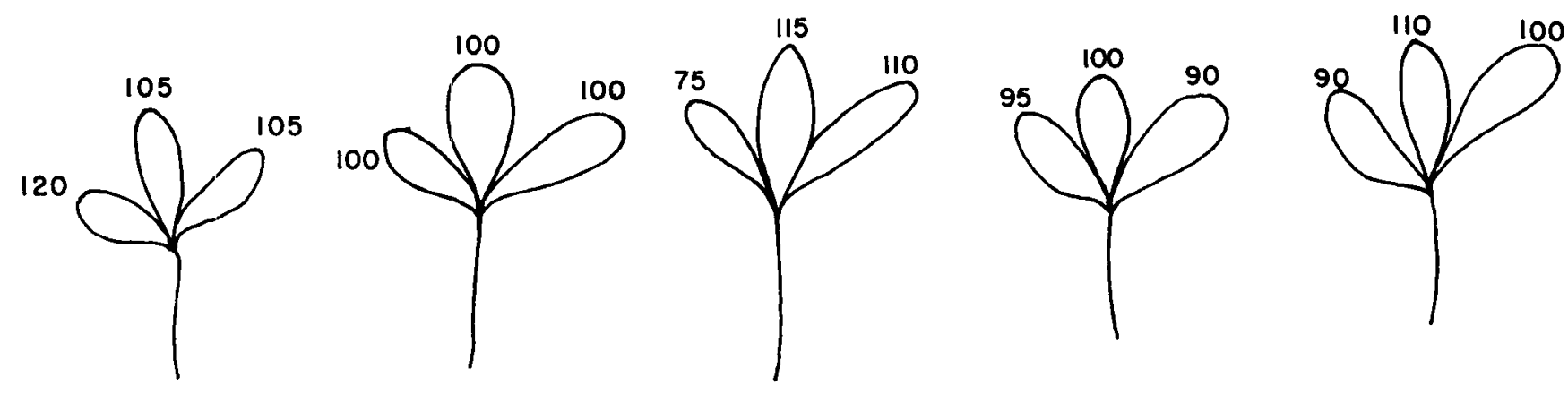

Figure 1

Exemples de la variation spatiale de la résistance stomatique observée sur la face supérieure d'une feuille de luzerne.

Examples of the spatial variation in stomatal diffusion resistance observed on the top side of a lucerne leaf. 
B. Variabilité dans les mesures de $\mathbf{r}_{\mathrm{s}}$ obtenues dans la strate supérieure de la végétation

1. Variabilité entre les feuilles d'une même plante (strate supérieure)

L'expérience précédente a été répétée sur plusieurs feuilles choisies dans la tranche supérieure de végétation et sur une même plante ; ceci induit des source supplémentaires d'hétérogénéité puisque l'inclinaison des feuilles diffère entre elles et que les feuilles testées, quoique éclairées, ne sont donc pas soumises à des conditions d'éclairement identique. Le but recherché à travers cette expérience est d'étudier la variabilité de la résistance stomatique $r_{s}$ sur différentes feuilles physiologiquement similaires et soumises à des conditions climatiques voisines.

La figure 2 donne les différentes valeurs de $r_{s}$ obtenues sur la face supérieure des feuilles pendant une trentaine de minutes, en milieu de journée de beau temps. Les feuilles

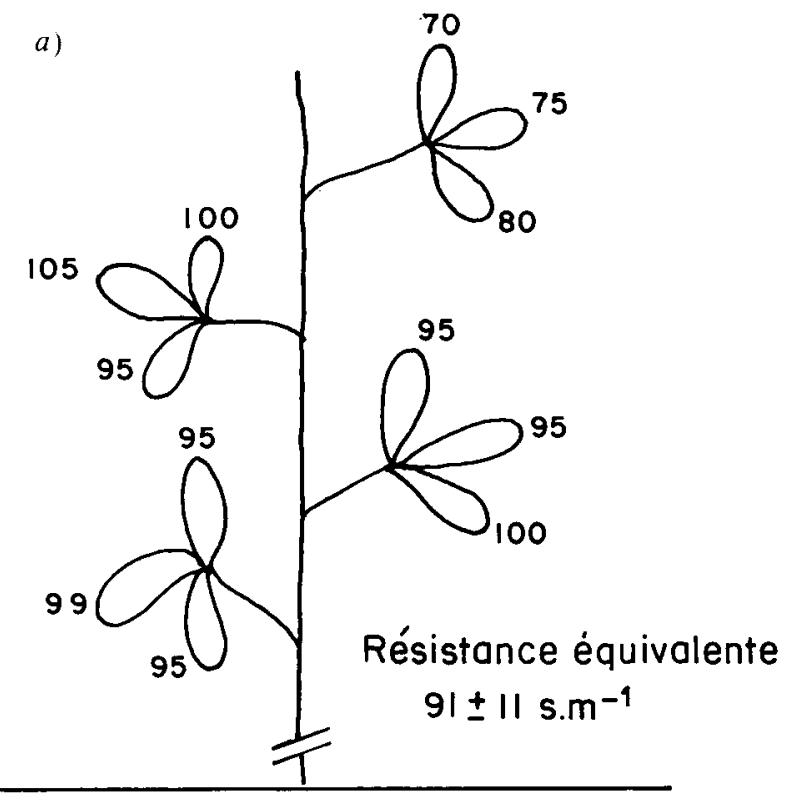

b)

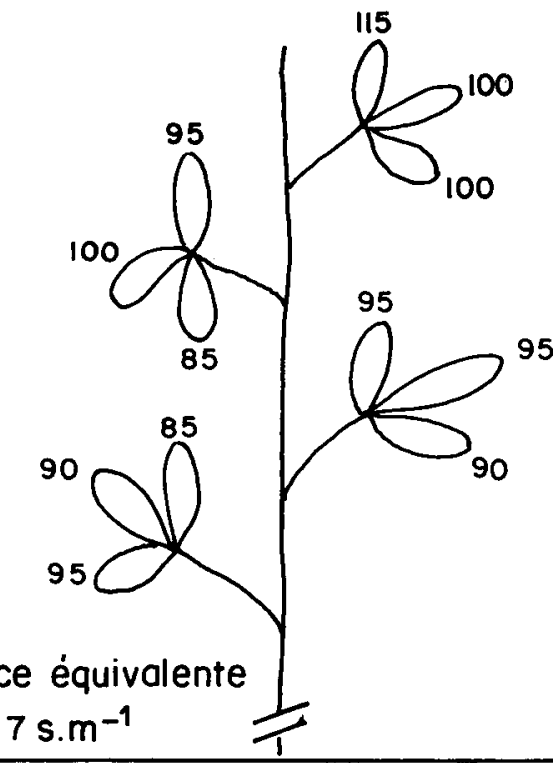

Figure 2

Exemples de la variation spatiale de la résistance stomatique observée dans la strate supérieure de la végétation sur la face supérieure des feuilles de luzerne.

Examples of the spatial variation in stomatal diffusion resistance observed on the top sides of lucerne leaves in the uppermost layer of vegetation. testées provenaient de 2 plantes choisies au hasard dans le champ mais ayant une structure identique, les feuilles ainsi choisies ayant une même position par rapport à la plante et au champ. On constate que les valeurs de $r_{s}$ obtenues ne semblent pas trop différentes au niveau d'une plante, ni d'une plante à l'autre ; en effet, la résistance équivalente de chaque échantillon ainsi que l'écart type sont très proches et pratiquement du même ordre de grandeur.

Il n'en va pas de même pour les résistances mesurées sur la face inférieure des feuilles dans les mêmes conditions expérimentales; en effet, les valeurs obtenues sont plus élevées sur la face inférieure et les résultats sont plus dispersés (fig. 3). Deux facteurs qui agissent dans le même sens peuvent être proposés pour expliquer ce comportement :

- les valeurs plus élevées sur la face inférieure sont dues à l'inégalité du nombre de stomates des 2 faces (FERRY \& WARD, 1959 ; DJAVANCHIR, 1971) ; en effet, DE PARCEVAUX (1972) trouve 58,6 stomates par $\mathrm{mm}^{2}$ pour la face supérieure des feuilles de luzerne et 46 pour la face inférieure.

- une différence d'éclairement reçu sur les 2 faces; en effet, même en pleine lumière, la face inférieure est toujours plus ombrée que la face supérieure.

Or les études concernant la relation entre éclairement reçu et résistance stomatique sur des feuilles de maïs, de sorgho et de tabac (TURNER, 1970) et sur des feuilles de luzerne (SHEEHY et al., 1979; KATERJI, 1982) montrent bien que les valeurs obtenues de résistance stomatique sont d'autant plus élevées et dispersées que le rayonnement reçu par les feuilles est de plus en plus faible ; ceci s'observe très nettement dès que l'éclairement devient inférieur à un certain seuil. On peut admettre donc que l'interaction entre les 2 causes précédentes, qui agissent dans le même sens, sont très certainement à l'origine de la différence du comportement observé entre les 2 faces.

\section{Etude de la variabilité entre les feuilles d'une même strate dans des conditions climatiques typiques}

Les exemples présentés dans les paragraphes précédents ont permis de cerner l'hétérogénéité des valeurs de résistance stomatique dans des cas particuliers (variation sur une même feuille, sur une même plante et surtout variation en conditions de beau temps). Nous avons alors étendu l'étude à toutes les conditions climatiques typiques rencontrées généralement au champ (temps variable, couvert, ciel dégagé). Le tableau 1 résume les résultats de l'étude entreprise pendant 3 ans au cours de laquelle on a réalisé, dans la strate supérieure, 10000 mesures de $r_{s}$ groupées en 975 échantillons, 489 pour la face supérieure et 485 pour la face inférieure. Chaque échantillon était composé en moyenne de 10 mesures de $r_{s}$ faites au hasard sur 10 feuilles de différentes plantes, mesures réalisées en $15 \mathrm{mn}$ environ. Pour cerner la variabilité de $r_{s}$, nous avons calculé le coefficient de variation (écart-type/moyenne) et les résultats de ces calculs ont été classés selon la face étudiée et le type de temps en 4 gammes de coefficient de variation (inférieur à $10,10-20,20-30$, supérieur à $30 \mathrm{p} .100$ ). On constate d'après le tableau 1 que, dans des conditions de temps stable (ciel dégagé, ciel couvert) et quelle que soit la face considérée, 68 p. 100 en moyenne des échantillons ont un coefficient de variation compris entre 0 et 20 p. 100 ; par contre, avec des conditions de temps variable (passage nuageux pendant une belle journée, apparition du soleil pendant une journée nuageuse), on ne retrouve que 40 à 50 p. 100 des échantillons dans cette gamme de variation. 


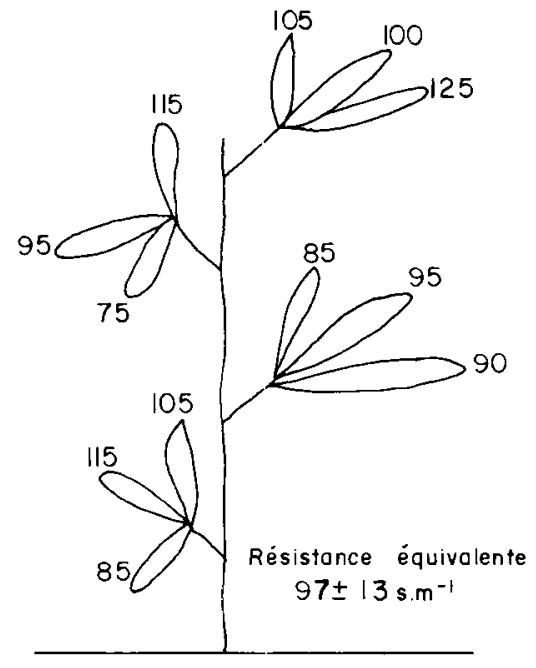

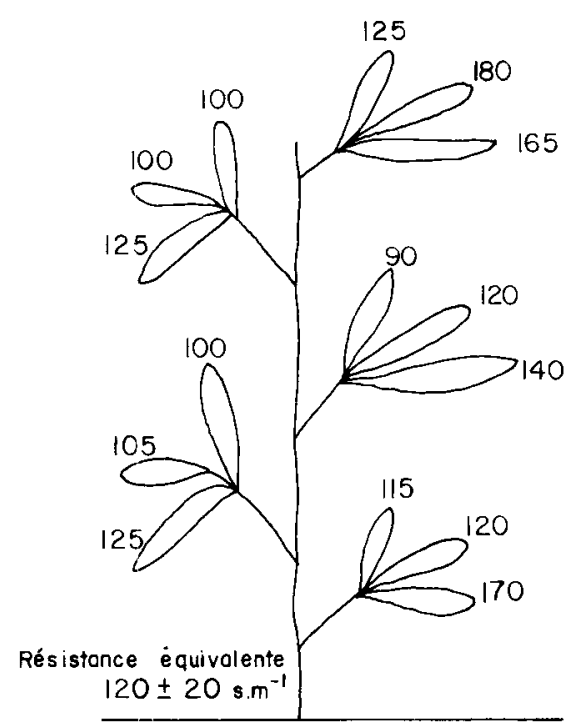

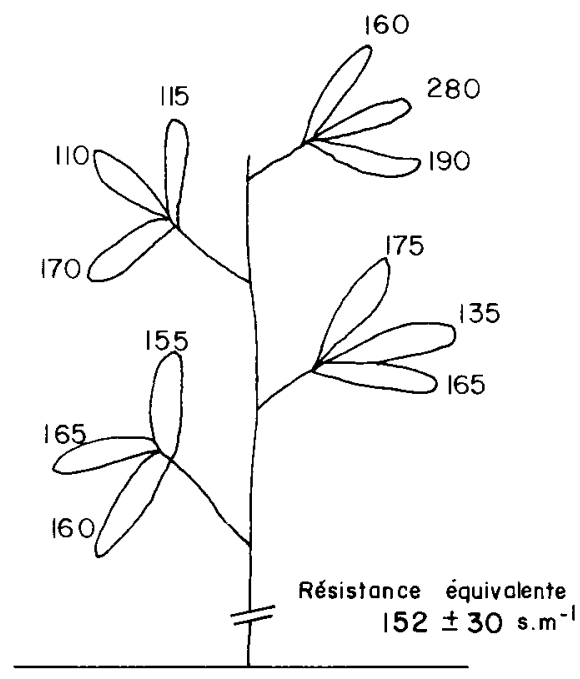

Figure 3

Exemples de la variation spatiale de la résistance stomatique observée dans la strate supérieure de la végétation sur la face inférieure des feuilles de luzerne.
Examples of the spatial variation in stomatal diffusion resistance observed on the bottom sides of lucerne leaves in the uppermost layer of vegetation.

TABLEAU 1

Coefficients de variation des échantillons de $r_{s}$ obtenus dans la strate supérieure de la végétation selon la face mesurée et le type de temps. Variation coefficients of $r_{s}$ samples obtained in the uppermost layer of vegetation according to side measured and weather type.

\begin{tabular}{|c|c|c|c|c|c|c|c|}
\hline & Coefficien & variation & $<10 \%$ & $10-20 \%$ & $20-30 \%$ & $>30 \%$ & Total \\
\hline \multirow{6}{*}{ 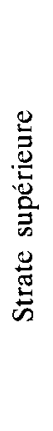 } & \multirow{3}{*}{ 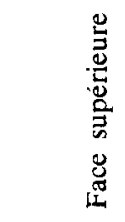 } & $\begin{array}{l}\text { ciel } \\
\text { clair }\end{array}$ & $5 \%$ & $65 \%$ & $97 \%$ & $100 \%$ & 201 \\
\hline & & $\begin{array}{l}\text { ciel } \\
\text { couvert }\end{array}$ & $6 \%$ & $70 \%$ & $98 \%$ & $100 \%$ & 166 \\
\hline & & $\begin{array}{l}\text { temps } \\
\text { variable }\end{array}$ & - & $37 \%$ & $89 \%$ & $100 \%$ & 122 \\
\hline & \multirow{2}{*}{ 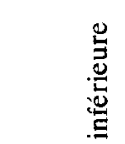 } & $\begin{array}{l}\text { ciel } \\
\text { clair }\end{array}$ & $5 \%$ & $64 \%$ & $96 \%$ & $100 \%$ & 201 \\
\hline & & $\begin{array}{l}\text { ciel } \\
\text { couvert }\end{array}$ & $4 \%$ & $71 \%$ & $98 \%$ & $100 \%$ & 163 \\
\hline & $\underset{\mathscr{E}}{\mathscr{E}}$ & $\begin{array}{l}\text { temps } \\
\text { variable }\end{array}$ & - & $51 \%$ & $90 \%$ & $100 \%$ & 121 \\
\hline
\end{tabular}

La dispersion relativement importante dans les conditions de temps variable peut être attribuée à la mise en régime de l'ouverture ou de !a fermeture des stomates, ces différences de régime pouvant être accentuées par le régime oscillatoire des mouvements stomatiques; en effet, bien que ces mouvements soient observés en conditions de milieu stables, il semble que ceux-ci prennent naissance ou sont amplifiés à l'occasion d'une perturbation de l'environnement (lumière, humidité de l'air) (SHIRAzI et al., 1976a, b ; AHO, 1981).

Une expérience faite au champ semble confirmer l'hypothèse précédente montrant bien, dans le cas d'une variation brusque de rayonnement, le rôle de ce paramètre sur la dispersion observée dans les mesures de $r_{\mathrm{s}}$. Le protocole de l'expérience était le suivant:

Pendant $10 \mathrm{mn}$, nous avons effectué des mesures de $r_{s}$ sur la face supérieure des feuilles du sommet de la végétation; nous avons vérifié par une cellule Lambda que ces feuilles recevaient le même éclairement (entre 890-1000 $\mathrm{Wm}^{-2}$ ). Puis nous avons divisé la parcelle étudiée en 2 parties ; sur l'une d'elles nous avons installé un écran artificiel, l'autre est restée sous éclairement normal.

Après $5 \mathrm{mn}$ d'ombre, nous avons effectué (fig. 4) alternativement, pendant $1 \mathrm{~h}$., 5 mesures de $\mathrm{r}_{\mathrm{s}}$ sur la face supérieure des feuilles éclairées et 5 mesures sur les faces supérieures des feuilles ombrées. On a vérifié au cours de l'expérience que les feuilles testées, soit à l'ombre, soit à la lumière, recevaient pratiquement le même rayonnement (70-80 $\mathrm{Wm}^{-2}$ à l'ombre, $890-1000 \mathrm{Wm}^{-2}$ à la lumière).

On vérifie bien alors (fig. 4) que les variations brusques du rayonnement conduisent à des valeurs de résistance plus élevées mais surtout plus dispersées que celles obtenues à la lumière.

\section{Variation verticale de la résistance stomatique}

Par rapport aux études précédentes, effectuées sur des feuilles d'une même strate et soumises à des conditions climatiques sensiblement homogènes, l'étude de la variation verticale de $r_{s}$ se fait sur des feuilles soumises, par leur 


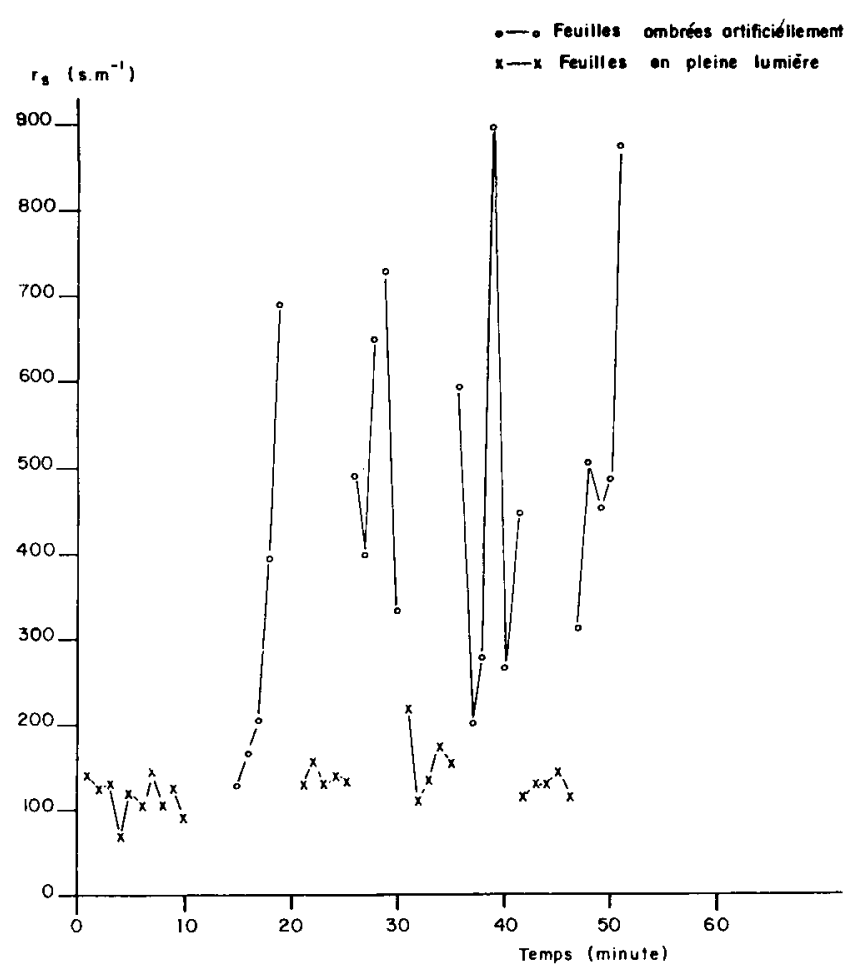

Figure 4

Effet d'un ombrage artificiel sur les valeurs obtenues de la résistance stomatique au cours du temps.

The effect of artificial shading on stomatal diffusion resistance values obtained over time.

position dans le couvert, à des facteurs climatiques différents et correspondant à un état physiologique également différent. Ces deux raisons doivent naturellement provoquer une source supplémentaire d'hétérogénéité, fonction du niveau considéré au sein du couvert (hétérogénéité supérieure à la base du couvert). A titre d'exemple et parmi les centaines de profils de $r_{s}$ obtenus au champ, nous présentons 2 profils obtenus pendant une journée de beau temps et une journée de temps variable, la végétation ayant été divisée en strates de $20 \mathrm{~cm}$ d'épaisseur. Soixante mesures de $r_{s}$ ont été faites dans chaque cas à raison de 20 mesures dans chaque strate comprenant 10 mesures sur les faces supérieures et 10 .mesures sur les faces inférieures des feuilles de la strate.
Si l'on considère le profil obtenu au cours d'une belle journée (fig. 5), on constate qu'au fur et à mesure que l'on s'éloigne du sommet, les valeurs des résistances stomatiques deviennent plus grandes et plus dispersées quelle que soit la face considérée. Pour ce qui concerne le profil obtenu au cours d'une journée de temps variable (fig. 6), on constate que les valeurs des résistances stomatiques obtenues dans tes strates inférieures de la plante ont le même comportement que par beau temps, c'est-à-dire qu'elles sont plus élevées et plus dispersées que dans la strate supérieure. Notons cependant que les mesures de $r_{s}$ obtenues dans la strate supérieure conduisent à des valeurs nettement plus hétérogènes par temps variable que par beau temps. Ceci rejoint les observations faites sur l'hétérogénéité des valeurs de $r_{s}$ en conditions de temps variable.

Pour interpréter le comportement des stomates dans les strates inférieures de la végétation, deux explications peuvent être avancées concernant leur comportement :

- D'une part, la réduction progressive de l'éclairement moyen de la strate lorsqu'on passe du sommet à la base de la végétation (DJAVANCHIR, 1971 ; MONTEITH, 1973), réduction qui s'accompagne d'une plus grande hétérogénéité des éclairements ponctuels.

- D'autre part, un accroissement de l'âge de la feuille depuis le sommet jusqu'à la base (BROwN \& ROSENBERG, 1970 ; IMPENS et al., 1967 ; TURNER, 1969 ; DJAVANCHIR, 1971) et un état de vieillissement assez hétérogène dans les basses couches.

Pour pouvoir tester l'importance relative de ces 2 explications, nous avons effectué pendant plusieurs journées des comparaisons entre 2 profils obtenus pendant $2 \mathrm{~h}$. successives : un profil obtenu au milieu du champ dans les conditions naturelles, c'est-à-dire sur des feuilles soumises à des conditions variables d'éclairement d'un niveau à l'autre, un autre réalisé au bord du champ, toutes les feuilles du profil quel que soit leur niveau, se trouvant en pleine lumière. A titre de démonstration, une comparaison entre les 2 types de profil précédents est présentée (fig. 7) ; elle suggère que les valeurs élevées et hétérogènes des résistances stomatiques obtenues dans les strates inférieures de la végétation sont plus une conséquence de la réduction progressive du rayonnement sur la plante (dans le cas d'une luzerne avant floraison) qu'un effet du vieillissement physiologique puisque le profil obtenu au bord du champ montre qu'il n'y a pas de variation nette de résistance stomatique selon la hauteur.

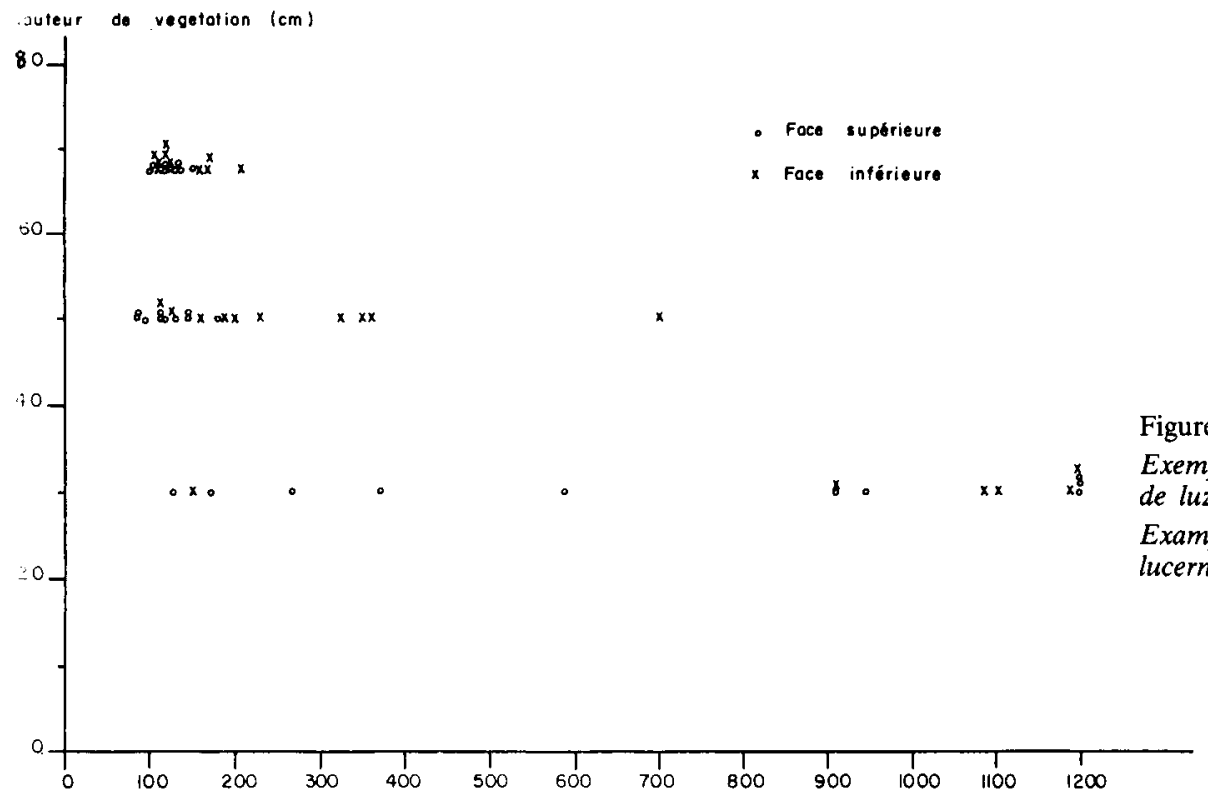



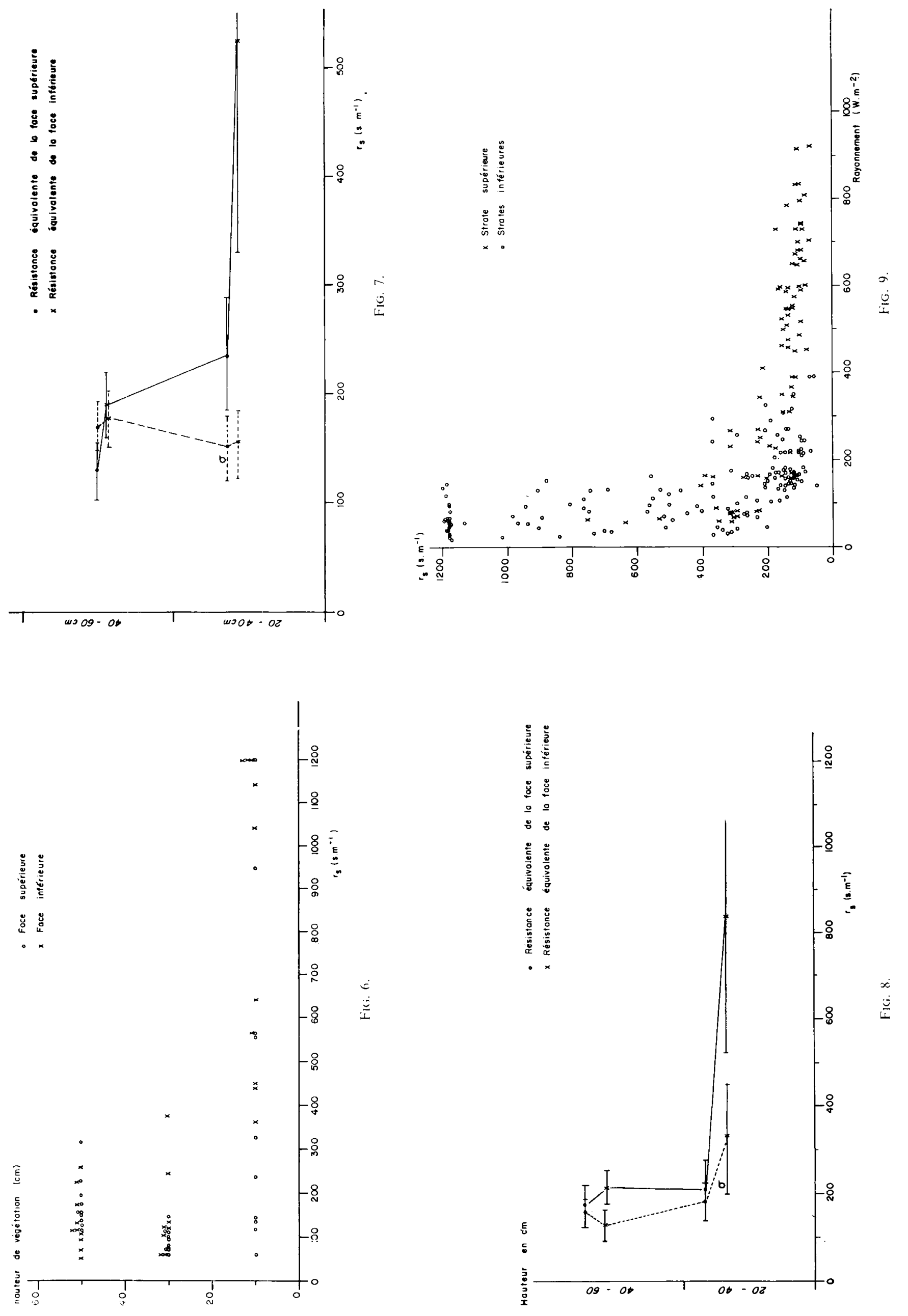
L'expérience précédente a été répétée dans les conditions suivantes: lors de la fauche de la culture, la parcelle expérimentale a été divisée en 2 parcelles plus petites ; la $1^{\text {re }}$ seule a été fauchée; au bord de la $2^{\mathrm{e}}$, non fauchée, les plantes sont celles qui se trouvaient avant la fauche au milieu du champ et n'ont donc pas subi d'effet de bordure. Nous avons effectué 2 profils de $r_{s}$, l'un au bord de cette parcelle et l'autre en milieu de parcelle. D'après la figure 8 , il apparaît nettement que les valeurs de résistance stomatique obtenues dans la strate inférieure deviennent significativement plus petites lorsque les feuilles reçoivent un rayonnement élevé.

Pour vérifier avec plus de précision ce dernier point, nous avons effectué, dans les différentes strates de la végétation (entre 0 et $60 \mathrm{~cm}$ ), des mesures simultanées de $r_{s}$ et d'éclairement. Les résultats sont présentés figure 9 , le rayonnement porté en abscisse a été mesuré par une cellule photoélectrique (Lambda) dans la gamme 300-1 $100 \mathrm{~nm}$, placée dans le même plan et au même point que la feuille testée.

$\mathrm{Si}$ on considère la figure 9 , toutes strates confondues, on observe les résultats classiques concernant la forme d'action de la lumière sur la régulation stomatique (TURNER, 1970). On peut en déduire approximativement une valeur critique du rayonnement $\left(400-500 \mathrm{Wm}^{-2}\right)$ au-delà de laquelle la résistance stomatique demeure plus ou moins constante. Remarquons aussi que tous les points expérimentaux de résistance obtenus dans la strate $0-40 \mathrm{~cm}$ se trouvent dans la

Figure 6

Exemple d'un profil de résistance stomatique observé sur une culture de luzerne au cours d'une journée de temps variable.

Example of a stomatal diffusion resistance profile observed on a lucerne crop during a day of variable weather.

Figure 7

Comparaison entre profils de résistance stomatique obtenus sous deux conditions d'éclairement au cours d'une journée de temps stable.

Les feuilles du profil sont soumises à des conditions d'éclairement différentes d'un niveau à l'autre.

Les feuilles du profil sont soumises au même éclairement. Comparison between stomatal diffusion resistance profiles obtained under two lighting conditions during a day of stable weather.

Profile leaves exposed to different lighting conditions from one level to the other.

Profile leaves exposed to the same lighting.

Figure 8

Comparaison entre profils de résistance stomatique obtenus sous deux conditions d'éclairement au cours d'une journée de temps variable.

Les feuilles $d u$ profil sont soumises à des conditions d'éclairement différentes d'un niveau à l'autre.

Les feuilles du profil sont soumises au même éclairement. Comparison between stomatal diffusion resistance profiles obtained under two lighting conditions during a day of variable weather.

Profile leaves exposed to different lighting conditions from one level to the other.

Profile leaves exposed to the same lighting.

Figure 9

Influence du rayonnement solaire incident (mesuré parallèlement à la feuille) sur la résistance stomatique obtenue dans les différentes strates de végétation.

Influence of incident solar radiation (measured parallel to the leaf) on stomatal diffusion resistance obtained in the different vegetation layers. gamme des rayonnements compris entre 0 et $400 \mathrm{Wm}^{-2}$ et que la majorité des valeurs de résistance mesurées dans la strate $40-60 \mathrm{~cm}$ appartiennent à la gamme $400-900 \mathrm{Wm}^{-2}$. Cette constatation explique le fait que les mesures de résistance obtenues dans la strate supérieure sont plus faibles et plus homogènes que celles obtenues dans les strates inférieures.

\section{DISCUSSION ET CONCLUSION}

Le problème de la représentativité des mesures de $r_{s}$ envisagé ici à plusieurs échelles (feuille, strate, plante entière) permet de dégager plusieurs résultats intéressants :

1. Lorsqu'on analyse la résistance stomatique de feuilles physiologiquement voisines (même stade de développement ou même strate), l'expérience montre que la variabilité observée dépend avant tout des conditions climatiques auxquelles sont soumises les feuilles testées. Ainsi plus les conditions climatiques sont stables, plus cette variabilité est faible (inférieure à $20 \mathrm{p} .100$ ); par contre, par temps variable, cette dispersion peut devenir beaucoup plus importante. Ce comportement, qu'on a pu provoquer au champ par un changement artificiel brusque de la lumière reçue par les feuilles, a déjà été observé dans les conditions du laboratoire et semble lié aux temps d'adaptation de la régulation et peut-être plus précisément au comportement oscillatoire des stomates (AHO, 1981). Notons quand même la faible variabilité observée dans les mesures de résistance que nous avons faites sur luzerne, variabilité faible en regard de ce que l'on a l'habitude de trouver sur d'autres cultures (CRUIZIAT et al., 1977). Deux explications peuvent être fournies qui se rapportent à la surface relativement réduite des feuilles de luzerne : d'une part, la petite surface des feuilles (de l'ordre de quelques $\mathrm{cm}^{2}$ ) permet une bonne représentativité de la résistance mesurée qui concerne une surface de l'ordre de grandeur de celles des folioles ; d'autre part, la petite surface des folioles ne favorise pas la formation de situations micrométéorologiques hétérogènes. Chez les plantes ayant de plus grandes feuilles (maïs, tournesol), ces conditions ne sont pas toujours réalisées, ce qui pourrait conduire à une hétérogénéité encore plus grande.

2. Lorsque la variabilité des résistances est envisagée à l'échelle de la plante entière, il apparaît nettement que les valeurs élevées et très dispersées de $r_{s}$ obtenues au fur et à mesure que l'on s'éloigne du sommet de la végétation puissent être liées à la réduction progressive et hétérogène de l'éclairement. L'âge physiologique ne semble pas jouer de rôle décisif dans notre étude puisque le même comportement a été observé sur des feuilles de la strate supérieure, donc physiologiquement jeunes, lors d'un passage nuageux par exemple (cf. fig. 4). Une explication peut être fournie à cet égard: elle est liée au fait que la coupe de luzerne intervient toujours en début de floraison; la plante se trouve encore en phase végétative et le processus de sénescence n'est pas encore entamé. Or TURNER (1969) a déjà montré, en se basant sur des mesures simultanées d'éclairement et de résistance stomatique effectuées sur des feuilles de maïs dont l'âge était variable, que l'âge de la feuille n'a pas d'influence apparente sur la résistance stomatique jusqu'à ce que la feuille commence à donner des signes de dégradation chlorophyllienne, comme le montre aussi notre étude sur le blé (KATERII, 1977).

Les résultats obtenus au cours de notre étude, et notamment ceux des figures 4 et 9 , font apparaître l'éclairement 
comme le seul facteur responsable de l'hétérogénéité observée dans le comportement stomatique. Cependant, d'autres facteurs (l'humidité relative de l'air, le vent, la teneur de l'air en $\left.\mathrm{CO}_{2}\right)$ peuvent aggraver cette hétérogénéité, bien que le rôle et l'importance de ceux-ci soient difficiles à démontrer dans les conditions naturelles.

Ainsi, on peut citer les travaux de AHO (1981) qui vérifient au laboratoire que la modification brusque de l'humidité de l'air sous faible éclairement peut, selon le cas (augmentation ou diminution de l'humidité), atténuer ou augmenter la variation cyclique ou l'hétérogénéité dans les mouvements stornatiques.

HEATH (1959) mentionne que chaque changement de pression se produisant dans l'épiderme de la feuille peut entraîner un mouvement passif des stomates. Or, au champ, les feuilles sont parfois pliées par le vent, ce qui peut aggraver dans certains cas l'hétérogénéité observée dans le comportement stomatique. Cependant, l'analyse de ce facteur de perturbation n'a pas encore été abordée à notre connaissance au laboratoire.

Le rôle des variations dans la teneur de l'air en $\mathrm{CO}_{2}$ sur le mouvement stomatique a été étudié au laboratoire par LOUGUET (1974) et l'auteur montre que les stomates qui se trouvent fermés à l'obscurité s'ouvrent lorsque la teneur de l'air en $\mathrm{CO}_{2}$ est supérieure à 4 p. 100 ; or la concentration en $\mathrm{CO}_{2}$ dans le végétal est susceptible de varier au cours de la journée et on peut se demander si c'est là un facteur important sur la résistance stomatique mesurée au sein du couvert.
En conclusion, les résultats présentés dans cette étude apportent des informations intéressantes sur la précision des résistances observées sur une culture de luzerne; ces informations permettront aux modélisateurs qui utilisent la résistance stomatique comme variable d'entrée et qui proposent de calculer en milieu naturel la photosynthèse ou l'évapotranspiration (MONTEITH, 1965 ; CHARTIER, 1970 ; PerRIER, 1976), de mieux cerner la signification et la précision des données calculées par ces modèles. Ainsi, les résultats de cette étude nous ont permis de préciser l'erreur affectant la résistance globale du couvert obtenue sur une culture de luzerne (KATERJI \& PERRIER, en préparation) lorsque celle-ci est déterminée selon la méthode décrite par MONTEITH $(1965,1973)$ par moyennage des conductances stomatiques des différentes tranches, pondérées par la densité de surface foliaire de la tranche. Cependant, il nous apparait intéressant de confirmer les résultats obtenus sur la luzerne par des expériences faites sur des cultures présentant des sources d'hétérogénéité plus importantes (cycle végétatif marqué par une succession de stades phénologiques, feuille de plus grande dimension); en effet, ces conditions qui favorisent les facteurs d'hétérogénéité physiques et physiologiques sont à l'origine de la dispersion observée dans les valeurs de résistance stomatique et pourront conduire à des résultats différents de ceux obtenus au cours de cette étude.

Reçu le 17 janvier 1983. Accepté le 5 mai 1983.

\section{RÉFÉRENCES BIBLIOGRAPHIQUES}

Aho N., 1981. Quelques aspects écophysiologiques des modes de réponse des plantes supérieures à la sécheresse atmosphérique: transpiration, photosynthèse, respiration. Thèse de Doct. d'état, Université de Paris VII, 188 p. + fig.

Barrs H. D., 1971. Cyclic variations in stomatal aperture, transpiration and leaf water potential under constant environmental conditions. Annu. Rev. Plant Physiol., 22, 223-236.

Berger A., 1971. La circulation de l'eau dans le système sol-plante. Etude de quelques résistances en relation avec certains facteurs $d u$ milieu. Thèse Doct, d'état, Université de Montpellier, $224 \mathrm{p}$.

Brown H. T., Rosenberg N. J., 1970. Influence of leaf age, illumination and upper and lower surface differences on stomatal resistance of sugar beet leaves. Agron. J., 62, 20-24.

Catsky J., Chartier Ph., Djavanchir A., 1973. Evolution diurne de la résistance stomatique et du déficit de saturation des feuilles. Conséquences sur la fixation de $\mathrm{CO}_{2}$. Ann. agron., 3, 287-305.

Chartier Ph., 1970. Etude de l'assimilation nette d'une culture couvrante. Thèse Doct. d'état, Univ. Paris XI, Orsay, 135 p.

Cowan I. R., 1972. Oscillations in stomatal conductance and plant functioning associated with stomatal conductance : observation and a model. Planta, 106, 185-219.

Cowan I. R., 1977. Stomatal behaviour and environment. $A d v$. Bot. Res., 4, 117-225.

Cruiziat P., Steimer J. M., Kobilinsky A., Malet A., Bodet C., 1977. Etude d'un modèle de la résistance stomatique de feuilles de tournesol. C. R. de fin d'étude D.G.R.S.T., Station de Bioclimatologie, Versailles, $52 \mathrm{p}$.

Demarly Y., 1957. Biologie et exploitation de la luzerne. Ann. Amélior. Plantes, 3, 247-272.

Djavanchir A., 1971. Mise au point d'une chambre de transpiration et son application a l'étude de la régulation stomatique. Thèse Doct. Ing., Université d'Orsay, 52 p. + fig.

Farquhar G. D., Cowan I. R., 1974. Oscillations in stomatal conductance. Plant Physiol., 54, 769-772.

Ferry J. F., Ward H. S., 1959. Fundamentals of plant physiology. MacMillan Company N.Y., 288 p.

Heath O. V. S., 1959. The water relation of stomatal cells and the mechanisms of stomatal movement. In: "Plant Physiology", Steward F. C. éd., Academic Press N.Y., 193-250.
Impens I. I., Steward D. W., Allen L. H., Lemon E. R., 1967. Diffusive resistances and transpiration rates from leaves in situ within the vegetative canopy of a corn crop. Plant Physiol., 42, 99104.

Johnson A., 1973. Oscillatory transpiration and water uptake of Avena plants. I. Preliminary observations. Physiol. Plant., 28, 4050.

Katerji N. B., 1977. Contribution à l'étude de l'évapotranspiration réelle du blé tendre d'hiver. Application à la résistance du couvert en relation avec certains facteurs du milieu. Thèse Doct. Ing., Univ. Paris VII, $121 \mathrm{p}+$ fig.

Katerji N. B., 1982. Etude et modélisation des transferts hydriques dans le système sol-plante-atmosphère. Thèse Doct. d'état, Univ. Paris VII, 199 p. + annexe 97 p.

Katerji N. B., Oulid-Aissa A., 1983. Problèmes posés par l'utilisation d'un poromètre à diffusion en milieu naturel. Agronomie, 3 (9), 933-938.

Kozlowski T. T., 1976. Water deficits and plant growth.

Vol. 1. Development, control and measurement, 1968, $390 \mathrm{p}$.

Vol. 2. Plant water consumption and response, 1968, $333 \mathrm{p}$.

Vol. 3. Plant response and control of water balance, 1972, $368 \mathrm{p}$.

Vol. 4. Soil water measurement, plant responses and breeding for drought resistance, $1976,383 \mathrm{p}$.

Academic press, London.

Louguet Ph., 1974. Les mécanismes du mouvement des stomates : étude critique des principales théories classique et moderne et analyse des effets du gaz carbonique sur le mouvement des stomates du Pelargonium $\times$ hortorum à l'obscurité. Physiol. veg., 12 (1), 53 81.

Meidner H., Mansfield T. A., 1968. Physiology of stomata. McGrow Hill Ld., 179 p.

Monteith J. L., 1965. Evaporation and environment. In : "The state and movement of water in living organisms ». Symp. Soc. for Exper. Biol. XIX, 205-234, Univ. Press Cambridge.

Monteith J. L., 1973. Principles of environmental physics. Arnold Ed. London., $241 \mathrm{p}$.

Parcevaux S. de, 1963. Transpiration végétale et production de matière sèche. Essai d'interprétation en fonction des facteurs du milieu. Ann. agron., 5, 655-742. 
Parcevaux S. de, 1972. Contribution à l'étude des échanges gazeux au niveau des feuilles. Rôle des résistances ou longueurs de diffusion en écophysiologie. Thèse Doct. d'état, Université d'Orsay, 86 p. + annexe.

Perrier A., 1976. Etude et modélisation des échanges de masse et d'énergie au niveau des couverts végétaux. Thèse Doct. d'état, Université de Paris VI, $236 \mathrm{p}$.

Sheehy J. E., Woodward F. I., Jones M. B., Windram A., 1979. Microclimate photosynthesis and growth of lucerne. I. Microclimate and photosynthesis. Ann. Bot., 44, 693-707.

Shirazi G. A., Stone J. F., Todd G. W., 1976a. Oscillatory transpiration in a cotton plant. I - Experimental characterization. $J$. exp. Bot., 27, 608-618.

Shirazi G. A., Stone J. F., Bacon C. M., 1976b. Oscillatory transpiration in a cotton plant. II - A model. J. exp. Bot., 27, 619-633.
Schoch P. G., 1972. Variation de la densité stomatique du Capsicum annuum $\mathrm{L}$. en fonction du rayonnement global. C. R. Acad. Sci., Paris, série D, 274, 2496-2498.

Slatyer R. O., 1967. Plant water relationships. Academic Press N.Y., 366 p.

Stocker O., 1961. Les effets morphologiques et physiologiques du manque d'eau sur les plantes. In : "Echanges hydriques en milieu aride ou semi-aride". Actes du colloque de Madrid, UNESCO, 69113.

Turner N. C., 1969. Stomatal resistance to transpiration in three controlling canopies, Crop Sci., 9, 303-307.

Turner N. C., 1970. Illumination and stomatal resistance to transpiration in three field crops. In : "Réponse des plantes aux facteurs climatiques». Actes du colloque d'Uppsala, UNESCO, 63-67. 